\title{
Lateralization of neural control for vocalization by the frog (Rana pipiens)
}

\author{
RICHARD H. BAUER \\ Middle Tennessee State University, Murfreesboro, Tennessee
}

\begin{abstract}
The hypothesis that anuran vocalization is lateralized was examined. Vocalization was induced by clasping the animal behind the forelimbs for $30 \mathrm{sec}$ in the morning and $30 \mathrm{sec}$ in the afternoon for 6 weeks. On the basis of the number of baseline vocalizations, four matched groups were formed ( $n=11)$ : nonoperates, sham operates, and animals receiving knife cuts in the left or right side just posterior to the brain areas that are thought to be the major vocalization generators. Following recovery, the number of vocalizations was recorded for 6 additional weeks. Vocaliza. tion was reduced significantly more by sections in the left side than in the right side, suggesting that one of the most basic relationships between the brain and vocal communication evolved very early and that natural language is based on previous adaptations in animals.
\end{abstract}

One view of natural language states that although human language is more complex than vocal communication by other animals, natural language evolved from previous adaptations in animals (for excellent reviews, see Lieberman, 1984; Pinker \& Bloom, 1990). At the other extreme, a contrasting view proposes that natural language is not a product of previous adaptations in animals but is a side effect of an increase in overall brain size (Chomsky, 1986; Pinker \& Bloom, 1990). Although no single experiment or observation is ever likely to resolve these complex issues, the position that language is based on previous adaptations in animals would be strengthened by demonstrating that there are basic similarities between humans and animals with small brains that evolved very early.

Greater involvement of the left hemisphere than of the right hemisphere in speech is one of the most well-known relationships between brain and behavior (see Caplan, 1987; Nass \& Gazzaniga, 1987). There was apparently no evidence that animal vocal communication was lateralized until Nottebohm $(1970,1971)$ demonstrated a lefthemispheric dominance for singing by birds. The left hemisphere is now known to be more important for bird song in several different species (for excellent reviews, see Nottebohm, 1980; Williams, 1990), suggesting that language is based on previous adaptations in animals.

Anurans appear to be the earliest vertebrates with true vocal cords (Vial, 1973), and anurans have several types of vocalization, some of which are apparently used to communicate with conspecifics (Aronson \& Noble, 1945; Kelley \& Tobias, 1989; Rand, 1988; Schmidt, 1976). For example, when a male mounts (clasps) another male or

\footnotetext{
I would like to thank $\mathrm{K}$. Barton for assistance in collecting the data, S. Amini for doing the histology, and D. Mitchell, J. Fuster, and C. McCandlish for critically reading the manuscript. This research was supported by the MTSU Faculty Research Program. Correspondence should be addressed to the author at Box 510, MTSU, Murfreesboro, TN 37132.
}

nongravid female, the mounted animal emits a series of calls, and, in response to the calls, the animal is released. However, if a male mounts a gravid female, no release calls are emitted, and clasping is maintained until the eggs have been deposited and fertilized (Aronson \& Noble, 1945; Kelley \& Tobias, 1989; Rand, 1988; Schmidt, 1976). Apparently, anuran males cannot readily discriminate between the sexes or between gravid and nongravid females, and vocalization communicates the necessary information. Mating calls are emitted by males during the breeding season to attract female conspecifics and, in some species, serve as territorial or agonistic signals to adjacent males (Rand, 1988).

Neurons in the anuran pretrigeminal area (PTA), which is located just anterior to the border of the tectum and cerebellum, project to the cranial nuclei which innervate the musculature involved in vocalization (larynx; Kelley \& Tobias, 1989; Schmidt, 1973, 1976, 1984; Schneider, 1988; Wetzel, Haerter, \& Kelley, 1985). On the basis of stimulation, lesion, recording, and anatomical studies, it has been suggested that the PTA and/or the surrounding area are the major generators of release and mating calls (Kelley \& Tobias, 1988; Schmidt, 1973, 1976; Schneider, 1988; Wetzel et al., 1985).

Although neural control of vocalization is lateralized in humans and songbirds, the possibility that vocalization by animals that evolved before birds is an asymmetrical brain function has apparently not been investigated. The purpose of the present study was to investigate whether anuran vocalization is also lateralized.

\section{METHOD}

\section{Subjects}

Adult male Rana pipiens of approximately $8 \mathrm{~cm}$ in length were obtained from a commercial supplier (W. A. Lemberger Co.). The animals were housed approximately 5 animals to a cage in a colony room maintained at $20^{\circ} \mathrm{C}$. The cages were plastic baskets covered with wire screen. Each cage had two $42 \times 28 \times 27 \mathrm{~cm}$ compartments 
interconnected with a galvanized pipe $15 \mathrm{~cm}$ in diameter. One compartment had approximately $2 \mathrm{~cm}$ of water; the other compartment was dry. The animals were maintained on a diet of crickets and/or worms. A full spectrum fluorescent light directly above each cage was on from 8:00 a.m. to 4:00 p.m. The fluorescent room lights were on from 6:00 a.m. to 6:00 p.m.

\section{Procedure}

Behavioral. Simulating clasping by holding Rana males with the thumb and forefinger just behind the forelimbs induces reliable release calls that are indistinguishable from the calls produced by normal clasping (Aronson \& Noble, 1945). Four days after the animals' arrival, calling was induced by simulating clasping for $30 \mathrm{sec}$ at approximately 9:00 a.m. and for $30 \mathrm{sec}$ at approximately 3:00 p.m., for 5 days a week for 6 weeks. On the basis of the calling rates during the 6-week baseline, four matched groups were formed ( $n=11$ ): (1) nonoperates, (2) sham operates, and (3) animals with left- or (4) right-side knife cuts at the border of the cerebellum and tectum. Following surgery, the animals were allowed 4 days to recover before release calls were recorded for 6 additional weeks using the same procedure that was used during the baseline period. The technician who elicited croaking was unaware of the animals' group membership.

Surgery and Histology. Nonoperates did not receive any treatments associated with the operation. Sham operates were anesthetized, the skin was retracted, and a hole was drilled in the skull above the tectal-cerebellar border. In addition to the procedures given to the sham operates, the other two groups received cuts through the total dorsal-ventral extent of the brain on either the left or right side, immediately in front of the cerebellum.

Hypothermic anesthesia was produced by immersion in an ice water $-10 \%$ alcohol solution for approximately $20 \mathrm{~min}$. Surgery was done under an operating microscope $(20 \times)$, with the animal's body covered with ice. A longitudinal incision was made along the midline, and the skin was retracted. A dental burr was used to drill a hole in the skull approximately $1.5 \mathrm{~mm}$ in the anterior-posterior dimension and from the midline to approximately $2.0 \mathrm{~mm}$ off the midline. Sections were made by inserting a fine-pointed scalpel blade perpendicular to the brain at the midline. The blade was lowered to the base of the skull and moved laterally, with the tip in contact with the base of the skull. The blade was moved to within $.25 \mathrm{~mm}$ of the dorsolateral edge. Since the anuran brain becomes narrower as one moves ventrally, a cut $.25 \mathrm{~mm}$ away from the dorsolateral surface will cut all of the tissue at deeper levels. The wound was cleaned and closed with surgical adhesive.

After the last day of testing the animals were sacrificed, and the brains were removed and fixed in $10 \%$ formaline. Each brain was mounted in parafin, and starting at the dorsal level of the brain stem, $10-\mu$-thick horizontal sections at 20 representative levels were mounted on glass slides and stained with hematoxyline and eosin.

\section{RESULTS}

The left side of Figure 1 shows the mean vocalization by each group during the last 2 baseline weeks. A 4 (groups) $\times 6$ (weeks) mixed analysis of variance (ANOVA) on the mean number of vocalizations per $30 \mathrm{sec}$ by each animal for each block of 5 days during the baseline revealed that there were no significant differences among the groups.

The mean number of vocalizations after the treatment per $30 \mathrm{sec}$ by each animal for each block of 5 days was analyzed by a 4 (groups) $\times 6$ (weeks) mixed ANOVA. There was a significant difference among the groups $[F(3,40)=14.30, p<.001]$, a significant increase across weeks $[F(5,200)=16.91, p<.001]$, and a significant interaction between groups and weeks $[F(15,200)=$ $3.92, p<.001$; Figure 1]. There was no significant difference in the mean number of vocalizations between the nonoperates and the sham operates. The two sectioned groups combined were significantly lower than the nonoperates $[F(1,31)=13.96, p<.001]$ and the sham operates $[F(1,31)=8.14, p<.008]$. Comparison of only the two sectioned groups showed that vocalization was reduced significantly more by sections on the left side than on the right side $[F(1,20)=8.45, p<.009]$. In both sectioned groups, there was a significant increase across weeks $[F(5,200)=14.43, p<.001]$, but the groups $\times$ weeks interaction was not statistically significant, indicating that some recovery occurred but that recovery was not reliably different in the two sectioned groups.

Figure 2 shows representative computer-generated sound waves from a frog with a knife cut in the left side (upper three waves) and a frog with a knife cut in the right side (lower three waves) that had preoperative and postoperative vocalization rates that were very close to their respective group means. The upper waves for each animal were taken in the 5th baseline week; the two lower waves for each animal were taken in the 4th postoperative week. As can be seen, the overall duration, amplitude, number of double glottal pulses, and discreteness of the pulses were severely reduced in the animal with a cut in the left side. In contrast, the duration, amplitude, number of double glottal pulses, and discreteness of the pulses by the animal with a cut in the right side were changed very little postoperatively.

Observations indicated that when vocalization was reduced after brain sections, the strong inspiration and expiration that normally occur during release calls were also reduced or eliminated. However, there was no indication that any aspect of breathing was impaired by damage to either side of the brain. All of the animals that were operated on had some vocal responses on the first few postoperative days.

Representative photomicrographs at a magnification of 40 times from an animal with a cut in the left side (left photograph) and an animal with a cut in the right side (right photograph) are shown in Figure 3. Microscopic examination of the slides showed that the left photomicrograph is, as near as can be determined, at the level at which the anterior-posterior dimension of the isthmic nuclei (IN) is the largest. The right photomicrograph is approximately $.40 \mathrm{~mm}$ ventral to the left photograph, or approximately $.20 \mathrm{~mm}$ above the most ventral aspect of IN. The distance between the posterior edge of IN and the knife cut is clearly greater on the left side than on the right side. This difference is not due primarily to differences in the position of the cuts but is due to the fact that as one moves ventrally, the posterior edge of IN moves posteriorly approximately $.20 \mathrm{~mm}$.

With the aid of a microscope, the distance between the posterior edge of IN at its broadest anterior-posterior dimension and the anterior edge of the cut-that is, the 


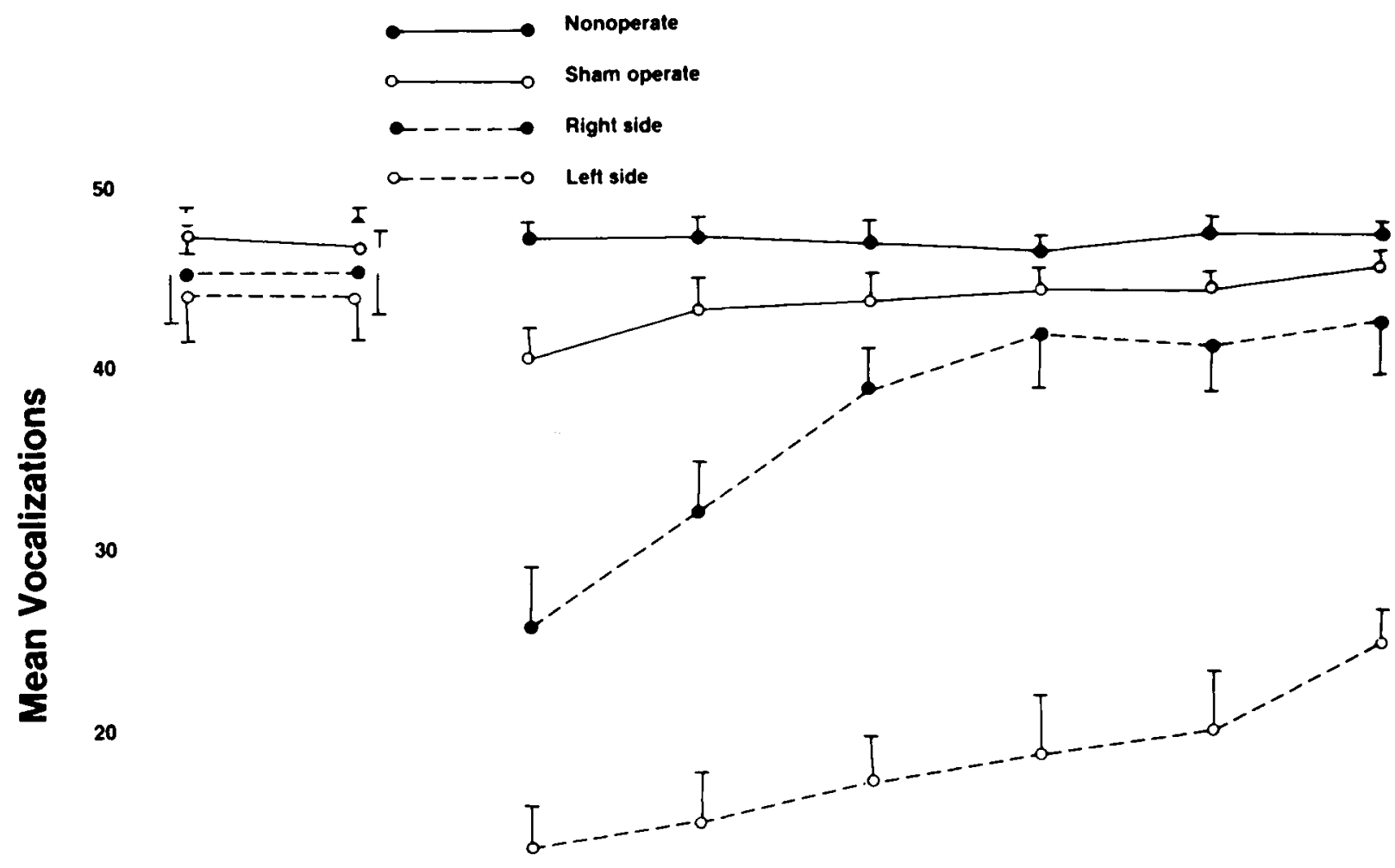

10

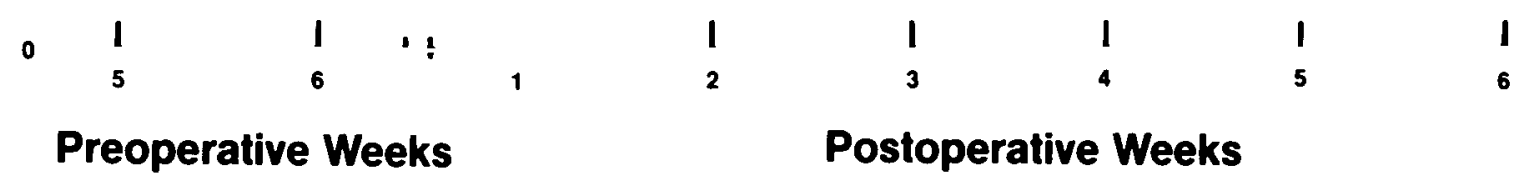

Figure 1. Mean vocalizations (per $30 \mathrm{sec}$ ) for the four groups during the last 2 weeks of baseline and during the 6 postoperative weeks. Brackets show standard errors of the means.

anterior-posterior position of the knife cut, was determined for each animal. A $t$ test showed that there was no significant difference between this distance on the left $(M=.55 \mathrm{~mm}, S E M=.022 \mathrm{~mm})$ and right $(M=$ $54 \mathrm{~mm}, S E M=.025 \mathrm{~mm}$ ) sides.

The distance between the posterior edge of IN at the level shown in the left photograph and the anterior edge of the knife cut ranked the animal at about the 7th most posterior position on the left side. The most posterior knife cut on either side was approximately $.10 \mathrm{~mm}$ posterior to the cut shown in the left portion of Figure 3. The distance between the posterior edge of IN at its largest anterior-posterior dimension and the anterior edge of the knife cut ranked the animal in the right photograph at about the 3rd most anterior position on the right side. The most anterior cut on either side was approximately $.10 \mathrm{~mm}$ anterior to the cut shown in the right portion of Figure 3.
The length of each cut from the midline to the most lateral aspect of the cut at the largest anterior-posterior dimension of IN was determined for each animal. The length of the cuts for each group was very similar (left side$M=1.40 \mathrm{~mm}, S E M=.044 \mathrm{~mm}$; right side $-M=$ $1.42 \mathrm{~mm}, S E M=.038 \mathrm{~mm}$ ), and a $t$ test showed that there was no significant difference between the lengths in the left and right sides.

Pearson product-moment correlations between the length of the cuts and the mean vocalization for the 6 postoperative weeks, and the anterior-posterior position of the cuts and the mean vocalization for the 6 postoperative weeks, were not statistically significant for either group separately or for both groups combined.

Microscopic examination revealed that in all the animals, the knife cuts extended through the total dorsalventral dimension. 

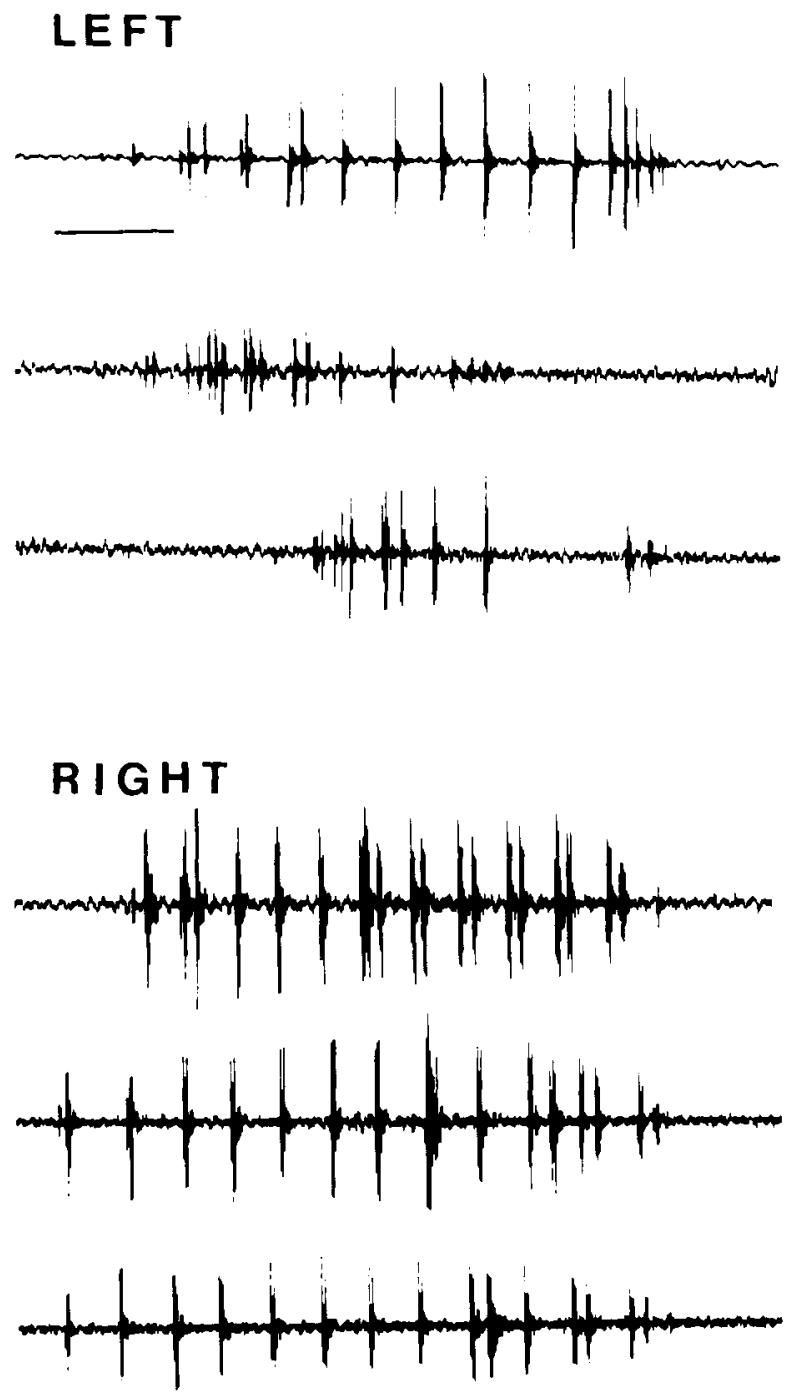

Figure 2. Representative computer-generated sound waves from an animal with a cut in the left side (upper three waves) and an animal with a cut in the right side (lower three waves). The upper wave for each animal are preoperative vocalizations; the two lower waves for each animal are postoperative vocalizations. The time line is 100 msec.

\section{DISCUSSION}

The present study appears to provide the first evidence for a left-hemispheric dominance for vocalization below birds. However, a number of important issues remain to be resolved. First, there are several different types of anuran calls (Rand, 1988), and it remains to be determined whether other anuran calls are lateralized. Anterior structures with ipsilateral connections to PTA are important for initiating mating calls, and mating calls are thought to have evolved from release calls (Kelley \& Tobias, 1989; Schmidt, 1973, 1976, 1984; Schneider, 1988; Wetzel et al., 1985), suggesting that mating calls may also be lateralized. The finding that many human and avian vocalizations are disrupted by left-hemispheric damage also suggests that the left side may play a more important role in other types of anuran calls. Second, the extent to which other anuran species are lateralized and whether the predominant pattern is a left-sided dominance remains to be determined. Some humans (approximately 5\%; Caplan, 1987) and one bird species of the nine species that have been examined (Williams, 1990; Williams, Crane, Hale, Esposito, \& Nottebohm, 1992) apparently have a right-sided dominance; occasionally, humans (probably less than 1\%; Caplan, 1987) and two avian species (Suthers, 1990) appear to have no dominant side, suggesting that some anurans will either have a dominant right side or have no dominant side. Third, the characteristics of anuran vocalization that are altered by damage to the left side have not been established. Damage to the lefthemispheric speech area reduces the quantity and quality of speech but has less effect on amplitude (Caplan, 1987), but in birds the emphasis has been almost exclusively on examining vocalization quality, intensity, and duration, and the number of vocalizations does not appear to have been examined (Nottebohm, 1980; Williams, 1990). Although the major emphasis of the present study was on the number of croaks, the sound waves (see Figure 2) and the experimenters' observations indicate that the quality and duration were also disrupted by sections in the left side. Another critical issue centers around whether the vocalization deficits produced by left-hemispheric damage in different species are based on common or different mechanisms. In the present and previous studies (Schmidt, 1973, 1976), the strong inspiration that normally precedes croaking was reduced by PTA damage, but in humans and birds brain damage that alters vocalization does not appear to alter inspiration associated with vocalization (Caplan, 1987; Lieberman, 1984; Williams, 1990). Thus, anurans may have at least one mechanism that differs from those in humans and birds. Damage to the left side alters vocalization quality in anurans, birds, and humans, suggesting that some comparable mechanisms are involved. Because of the complexity of the vocalization mechanisms, determining whether the same or different mechanisms are involved in different species is likely to be a difficult task.

Several factors indicate that the differences in vocalization produced by sectioning the left or right side were not due to differences in the position and/or the size of the sections. First, the blade was lowered to the base of the skull and moved laterally, so there was a very clear reference for the dorsal-ventral dimension. Second, the sections were made between the cerebellum and tectum, which provides a very clear reference, and there was no significant difference between the anterior-posterior position of the sections in the left and right sides. Third, there was no significant difference in the length of the cuts in the left and right sides. Fourth, the nonsignificant correlations between the postoperative vocalizations and the anterior-posterior position and the length of the sec- 


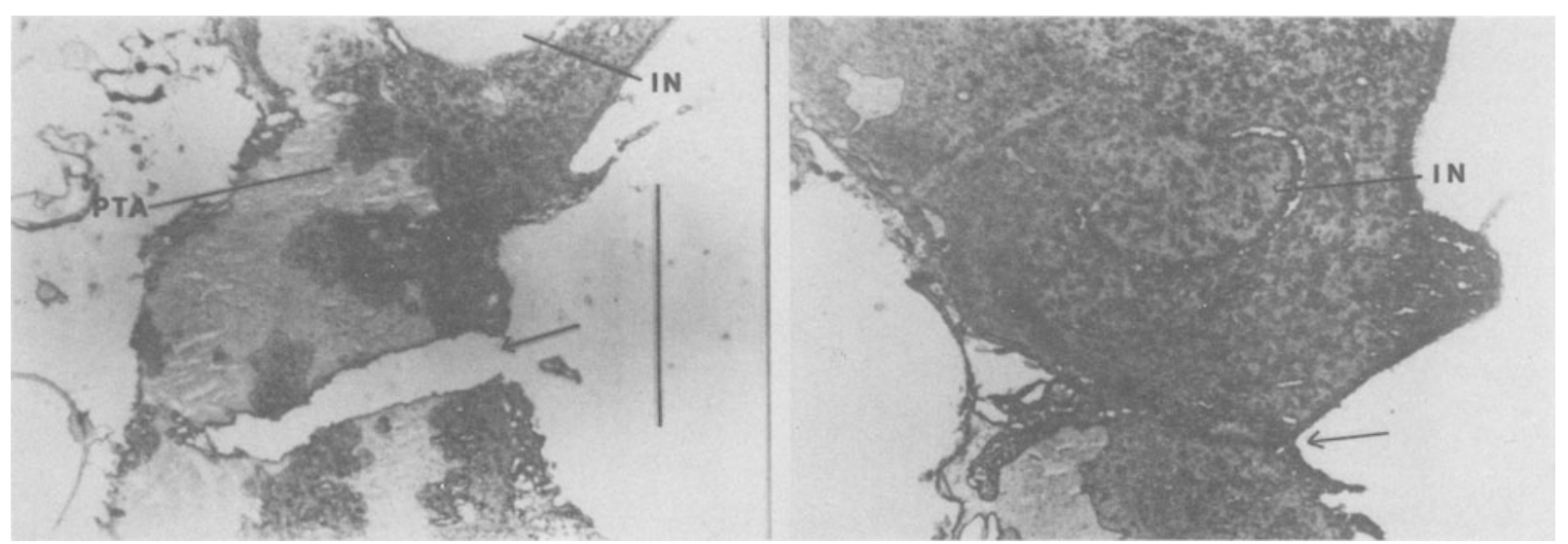

Figure 3. Representative horizontal sections in the left side (left photograph) and the right side (right photograph) showing cuts (arrows) posterior to the pretrigeminal area (PTA) and the isthmic nuclei (IN). The scale bar for both photomicrographs is .5 mm long.

tions indicate that the reduction in vocalization was not associated with the position or length of the sections. Finally, if the position or length of the sections were dissimilar within each group or between the two groups, the standard errors of the means for vocalization would be expected to be larger for sections in one side than in the other and larger postoperatively than preoperatively. As shown in Figure 1, the preoperative and postoperative standard errors were similar for sections in the left and right sides.

Although a search for the brain areas involved in anuran vocalization began a century ago (see the references in Aronson \& Noble, 1945), the area(s) and pathway(s) of the neural generator(s) for vocalization are still not precisely known. Without knowing the specific area(s) of the neural generator, attempting to demonstrate brain asymmetry for vocalization by destroying specific areas is obviously difficult. Furthermore, destroying progressively smaller areas makes it progressively more difficult to demonstrate that the damage is comparable on both sides. For these reasons, at the present time, cutting or destroying relatively large portions of the brain appears to be a more satisfactory way of investigating anuran lateralization of vocalization. In addition, knowledge that a more important role is played by the left side than by the right side would be expected to be valuable for specifying the location of the neural generators.

The finding that at least some components of vocal communication exhibit lateralization in Rana pipiens is likely to have important implications for behavioral neuroscience. For example, learning plays a significant role in human speech and bird song (Caplan, 1987; Nass \& Gazzaniga, 1987; Nottebohm, 1970, 1971, 1980; Williams, 1990), but there is apparently no evidence that learning plays a role in anuran vocal communication. Thus, contrary to previous speculations (Nottebohm, 1970), both learned and unlearned vocalizations appear to be lateralized.

Another interesting possibility is that lateralization of anuran vocalization is a primordial characteristic. Am- phibians are thought to represent an evolutionary transition from an aquatic to a terrestrial habitat, and the brain structures of anurans and terrestrial vertebrates are homologous (Herrick, 1933). The essential topography of the anuran forebrain, midbrain, and hindbrain, and the circuitry within these major divisions, have been reiterated in successive species throughout the entire vertebrate evolutionary scale (Caviness, 1987). In addition, there is a continuity in the peripheral structures, sound characteristics, and functions of vocal communication from anurans to humans (Lieberman, 1984). Results of the present study add to the list of similarities between animal and human vocal communication and suggest that one of the most basic relationships between the brain and vocal communication evolved very early. The similarities between animals and humans also supports the position that natural language is a more complex elaboration of vocal communication that evolved earlier in nonhuman animals. The similarities between frogs and humans becomes even more striking when one considers that anurans evolved at least 170 million years ago and were apparently one of the first groups to develop vocal communication (Vial, 1973).

\section{REFERENCES}

ARonson, L. R., Noble, G. K. (1945). The sexual behavior of anura: 2. Neural mechanisms controlling mating in the male leopard frog, Rana pipiens. Bulletin of the American Museum of Natural History, 86, 83-139.

CAPLAN, D. (1987). Neurolinguistics and linguistic aphasiology. New York: Cambridge University Press.

CAviness, V. S., JR. (1987). Neurogenetics and behavior related to higher brain functions. In V. B. Mountcastle, F. Plum, \& S. Geiger (Eds.), Handbook of physiology. Section I: The nervous system. Volume 5: Higher functions of the brain, Part / (pp. 109-135). Bethesda, MD: American Physiological Society.

СномSKу, N. (1986). Knowledge of language: Its nature, origin, and use. New York: Praeger.

Herrick, C. J. (1933). The amphibian forebrain: VIII. Cerebral hemispheres and pallial primordia. Joumal of Comparative Neurology, 58. 737-759.

Kelley, D. B., \& ToBiAs, M. L. (1989). The genesis of courtship song 
Cellular and molecular control of sexually differentiated behavior. In T. J. Carew \& D. B. Kelley (Eds.), Prospectives in neural systems and behavior (pp. 175-194). New York: Wiley.

Lieberman, P. (1984). The biology and evolution of language. Cambridge: Harvard University Press.

Nass, R. D., \& Gazzaniga, M. S. (1987). Cerebral lateralization and specialization in human central nervous system. In V. B. Mountcastle, F. Plum, \& S. P. Geiger (Eds.), Handbook of physiology. Section 1 : The nervous system. Volume 5: Higher functions of the brain, Part 2 (pp. 701-761). Bethesda, MD: American Physiological Society.

Noттевонм, F. (1970). Ontogeny of bird song. Science, 167, 950-956.

Nоттевонм, F. (1971). Neural lateralization of vocal control in a passerine bird: I. Song. Journal of Experimental Zoology, 177, 229-262.

Noттевонм, F. (1980). Brain pathways for vocal learning in birds: A review of the first 10 years. In J. M. Sprague \& A. N. Epstein (Eds.), Progress in psychobiology and physiological psychology (pp. 85-124). New York: Academic Press.

Pinker, S. , \& BloOM, P. (1990). Natural language and natural selection. The Behavioral and Brain Sciences, 13, 707-784.

RAND, A. S. (1988). An overview of anuran acoustic communication. In B. Fritzsch, J. J. Ryan, W. Wilczynski, \& T. E. Hethington (Eds.), The evolution of the amphibian auditory system (pp. 415-431). New York: Wiley.

Schmidt, R. S. (1973). Central mechanisms of frog calling. American Zoologist, 13, 1169-1177.
ScнміDт, R. S. (1976). Neural correlates of frog calling: Isolated brainstem. Journal of Comparative Physiology, 108, 99-113.

Schмidt, R. S. (1984). Neural correlates of frog calling: Preoptic area trigger of "mating calling." Journal of Comparative Physiology, 154, 847-853.

SCHNeider, H. (1988). Peripheral and central mechanisms of vocalization. In B. Fritzsch, J. J. Ryan, W. Wilczynski, \& T. E. Hethington (Eds.), The evolution of the amphibian auditory system (pp. 537558). New York: Wiley.

SUTHERS, R. A. (1990). Contributions to birdsong from the left and right sides of the intact syrinx. Nature, 347, 473-477.

VIAL, J. L. (Ed.) (1973). Evolutionary biology of anurans. Columbia: University of Missouri Press.

Wetzel, D. M., Haerter, U. L., \& Kelley, D. B. (1985). A proposed neural pathway for vocalization in South African clawed frogs. Xenopus laevis. Journal of Comparative Physiology, 157, 749-761.

Williams, H. (1990). Bird song. In R. P. Kesner \& D. S. Olton (Eds.), Neurobiology of comparative cognition (pp. 77-126). Hillsdale, NJ: Erlbaum.

Williams, H., Crane, L. A., Hale, T. K., Esposito, M. A., \& Notteвонм, F. (1992). Right-side dominance for song control in the zebra finch. Journal of Neurobiology, 23, 1006-1020.

(Manuscript received September 8, 1992; revision accepted for publication March 17, 1993.) 